\title{
Donor CSF3R with the rs3917980A/G or G/G genotype is correlated with better leukemia-free survival after allogenic hematopoietic stem cell transplantation
}

\author{
XunHong $\mathrm{Cao}^{1}$, Yan Hong ${ }^{1}$, XingXing $\mathrm{Yu}^{1}$, LanPing Xu${ }^{1}$, XiaoHui Zhang ${ }^{1}$, Yu Wang ${ }^{1}$, \\ KaiYan Liu ${ }^{1}$, XiaoJun Huang ${ }^{1}$, YingJun Chang ${ }^{1}$, XiangYu Zhao ${ }^{1}$, and XiaoSu Zhao ${ }^{1}$ \\ ${ }^{1}$ Peking University People's Hospital
}

July 3, 2020

\begin{abstract}
Polymorphisms in the granulocyte colony-stimulating factor receptor gene (GCSFR, CSF3R) have been reported to be associated with peripheral blood stem cell enrichment and hematological diseases. The aim of our study was to investigate the effects of donor CSF3R allelic polymorphisms on the outcomes of allogeneic stem cell transplantation. A total of 273 patients who were diagnosed with hematological diseases and treated with allogeneic hematopoietic stem cell transplantation(allo-HSCT) were enrolled in this study. Single-nucleotide polymorphisms in CSF3R were genotyped by targeted next-generation sequencing. There were six types of CSF3R genotypes with percentages over 1\%. LFS and OS analyses showed that recipients receiving grafts from healthy donors with an rs $3917980 \mathrm{G} / \mathrm{G}$ or A/G genotype had higher LFS rates than those receiving grafts from donors carrying an rs22754272 T/C genotype and the double negative group $(\mathrm{p}=.036)$ Univariate analysis showed that donor CSF3R with the rs2275472 T/C genotype was associated with higher transplantation-related mortality(TRM) rates (HR=3.77, 95\% CI: 1.416-9.631, $\mathrm{p}=.004)$ and higher rates of leukemia-free survival(LFS) (HR=2.523; 95\% CI: 1.032-6.13, $\mathrm{p}=.026)$. In addition, donor CSF3R with the rs3917980G/G or A/G genotype was associated with better overall survival(OS) rates (HR=0.512, 95\% CI: $0.244-1.017, \mathrm{p}=.047)$. Our findings demonstrate the important prognostic value of genetic variations in donor CSF3R to predict clinical outcomes in patients undergoing allo-HSCT.
\end{abstract}

\section{Keywords: CSF3R; allo-HSCT; SNP}

\section{Introduction}

Allogeneic hematopoietic stem cell transplantation (allo-HSCT) has become an important and accepted therapeutic treatment for malignant disorders. Hematopoietic stem cells can be obtained from bone marrow through multiple aspirations or alternatively from peripheral blood after stimulation with granulocyte colonystimulating factor (G-CSF). G-CSF has been indicated to promote the mobilization and differentiation of hematopoietic stem cells and is now a widely used scheme in HSCT [1]. However, the mobilization efficiency of G-CSF varies individually; that is, poor mobilization occurs in $25 \%$ of patients with hematological malignancies and in $10 \%-20 \%$ of healthy donors ${ }^{[2]}$.

G-CSR is a highly specific receptor that binds G-CSF and induces a series of signaling cascades, leading to different biological events ${ }^{[3,4]}$. Genomic sequencing of the CSF3R locus has identified many intronic polymorphisms and four synonymous coding mutations ${ }^{[5]}$, therefore discrepancies in the CSF3R gene, whether caused by SNPs or mutations, that have been proven to be associated with an abnormal response to GCSF treatment ${ }^{[6,7,8]}$. However, most current studies focus on the effect of CSF3R polymorphisms on the occurrence or progression of disease in patients ${ }^{[9]}$. Donor-derived hematopoietic stem cells are the products responding to G-CSF treatment. Bahar et al used PCR-RFLP technology to study whether differences in 
CSF3R SNP loci of healthy donors and hematological tumor patients would affect the enrichment of peripheral blood CD34 ${ }^{+}$cells, and the results showed that different SNP loci have different mobilization efficiencies for different individuals. Among them, healthy donors carrying the rs3918018 A/A genotype had significantly less efficient CD34 + collections in the first two attempts than donors carrying the G/G genotype [10]. Since the number of CD34 molecules is related to cell implantation, reconstruction and other events post allo-HSCT and CSF3R polymorphism may directly affect the prognosis of transplantation, whether the clinical prognosis of patients after HSCT will be affected by donor CSF3R polymorphisms has not yet been elucidated. Thus, the aim of the study was to screen CSF3R SNPs and therefore explore whether donor CSF3R SNPs can affect the clinical outcomes of patients post allo-HSCT.

\section{Materials and methods}

\subsection{Patients}

We performed a retrospective observational study to investigate the impact of donor CSF3R polymorphisms on the clinical outcomes of acute leukemia patients who underwent HLA-matched sibling HSCT or unmanipulated haploidentical allo-HSCT at the Peking University Institute of Hematology from April 2014 to April 2015. A total of 273 patients between the ages of three to sixty-two years old were enrolled in the present study, and all cases were diagnosed based on the World Health Organization diagnosis criteria. The study was conducted in accordance with the Declaration of Helsinki and was approved by the Institutional Review Board of Peking University.

All patients provided informed consent for the treatment under a protocol reviewed and approved by the Peking University Institute of Hematology. The patient and transplantation characteristics are summarized in Table 1 .

\subsection{Sequencing of CSFR single nucleotide polymorphisms (SNPs)}

Sequencing was performed using the Ion Torrent platform. CSF3R gene exon region and exon-intro junction region primers were designed by Ampliseq design. The Ion Ampliseq Library Kit, 2.0(Thermo Fisher) was used for library construction. The Ion PGM Hi-Q OT2 Kit and Hi-Q Sequencing $200 \mathrm{Kit}$ reagents were used to perform sequencing. Then, sequencing BAM files and variant caller files were acquired. We then performed data screening and judged the data quality based on mutation frequency, sequencing quality value, forward and reverse sequencing depth and chain bias. Then, SNP sites were screened by population frequency, mutation type and mutation function.

\subsection{Conditioning regimen and stem cell collection}

All patients received a myeloablative conditioning regimen (MAC) without in vitro T-cell depletion. The conditioning regimens used in our center were based on BU and total body irradiation (TBI). For patients who received haploidentical HSCT, the conditioning regimen was modified to include the following: BUCY+ATG (thymoglobulin) consisting of cytarabine $\left(4 \mathrm{~g} / \mathrm{m}^{2}\right.$ per day in haploidentical HSCT) intravenously on days -10 to -9 ; busulfan $(3.2 \mathrm{mg} / \mathrm{kg}$ per day, intravenously on days -8 to -6$)$; cyclophosphamide $(\mathrm{CY}, 1.8 \mathrm{~g} / \mathrm{m} 2$ per day) intravenously on days -5 to -4 ; Me-CCNU $(250 \mathrm{mg} / \mathrm{m} 2)$, orally once on day -3 ; and thymoglobulin (ATG, Sang Stat, Lyon, France; $2.5 \mathrm{mg} / \mathrm{kg}$ per day) intravenously for 4 consecutive days from days -5 to -2. Patients undergoing HLA-matched sibling donor HSCT received hydroxycarbamide $(80 \mathrm{mg} / \mathrm{kg})$ orally on day -10 and a lower dose of cytarabine ( $2 \mathrm{~g} / \mathrm{m} 2$ per day) on day -9 , but otherwise, these patients followed an identical regimen to haploidentical patients, without ATG. The TBI-based conditioning regimen was composed of the following: TBI (770 cGy for a single dose) on days -6 and cyclophosphamide (CY, $1.8 \mathrm{~g} / \mathrm{m} 2$ per day) intravenously on days -5 to -4 ; and Me-CCNU $(250 \mathrm{mg} / \mathrm{m} 2)$, orally once on day -3 . ATG was used for patients who received haplo-HSCT, and the dosage and time were consistent with the above description. All subjects received fresh granulocyte colony-stimulating factor (G-CSF)-mobilized bone marrow (BM) and peripheral blood cells. Patients also received prophylactic drugs to prevent infection by fungi and viruses.

\subsection{GVHD prophylaxis}


The prophylaxis for acute GVHD (aGVHD) was composed of cyclosporine, mycophenolate mofetil (MMF), and short-term methotrexate (MTX). The discontinuation time of MMF was different according to the transplantation type: (1) MMF was withdrawn when (White blood cells, WBCs ) engrafted in patients who received matched related transplantation (MRD) within 30 days post-HSCT; and (2) MMF was withdrawn on day 45 post-HSCT in patients who received mismatched related/haploidentical transplantation.

\subsection{Definitions and endpoints}

Neutrophil engraftment was defined as an absolute neutrophil count of $0.5 \times 10^{9} / \mathrm{L}$ or more for 3 consecutive days. Platelet engraftment was defined as a platelet count of $20 \times 10^{9} / \mathrm{L}$ or more for 7 consecutive days without transfusion. Acute and chronic GVHD were diagnosed and graded according to established criteria. Risk stratification was differentiated into two groups: AML and ALL in first or second remission (CR1 or CR2), chronic myeloid leukemia (CML) in the first or second chronic phase (CP1 or CP2), and MDS with refractory anemia with or without ringed sideroblasts were defined as the standard-risk group, and other malignant diseases were defined as the high-risk group. Overall survival (OS) times were measured from the date of HSCT until death from any cause. Leukemia-free survival (LFS) was defined as the time from transplantation to relapse or death from any cause. Surviving patients were censored on the date of their last follow-up. Transplantation-related mortality (TRM) was defined as death after allo-HSCT without disease progression or relapse. Relapse was defined by the appearance of morphological evidence of the disease obtained from testing samples from the peripheral blood, bone marrow, or extramedullary sites or by recurrence and the sustained presence of pretransplantation chromosomal abnormalities.

\subsection{Statistical analyses}

The Mann-Whitney U test was used to compare continuous variables, and the chi-square test or Fisher exact statistic was used to compare categorical variables. Cumulative incidence curves were used in a competing risk setting, with TRM related as a competing event, to calculate relapse probabilities. The probabilities of LFS and OS were estimated with the Kaplan-Meier method. All variables in Table 1 and Table 2 were included in the univariate analysis. Only variables with a $\mathrm{P}<.10$, including $\mathrm{T}$ cells from donors, PLT, rs3917980 and rs2275472 in CSF3R SNPs and disease risk, were included in a Cox proportional hazards model with time-dependent variables. $\mathrm{P}$ values were based on 2-sided hypothesis tests. The alpha level was set at .05. All data analyses were performed with the R Foundation for Statistical Computing 3.5.3 using the packages cmprsk, survival, surviminer.

\section{Results}

\subsection{Clinical data}

The demographic and clinical data of 273 patients post all-HSCT are listed in Table 1 . The average patient age at the time of the transplant was 29.71 years-old. The M:F sex ratio was 171 (62.6\%):102 (37.4\%). Acute myeloid leukemia, affecting $156(57.1 \%)$ patients, acute lymphoid leukemia, affecting 63 (23.1\%) patients, and myelodysplastic syndrome, affecting $33(11.9 \%)$ patients, were the most frequently found diseases. Among the patients, 254 had a standard risk status, and 19 had a high-risk status. At the time of allo-HSCT, 256 patients were in complete remission (CR), 2 were in partial remission, and 10 had disease activity. All patients received peripheral blood as well as bone marrow cells as their source of hematopoietic stem cells. Only $60(22.0 \%)$ samples were HLA-matched from their relatives. A total of 146 (53.5\%) patients developed acute GVHD, and 54 (19.8\%) patients were found to have a chronic form. As of April 2020, fifty (18.3\%) had relapsed, forty-three (15.8\%) finally developed transplantation-related mortality, and $188(67.8 \%)$ remained alive. The median follow-up time was 1799 days. The overall survival rate at five years was $32.2 \%$, whereas the leukemia-free survival rate was $32.7 \%$.

\subsection{Frequency of polymorphisms}

We analyzed 273 healthy donors for polymorphisms of the CSF3R gene, and the results showed that there are mainly six SNPs in CSF3R, which can be listed as rs2275471, rs78861150, rs3917981, rs3917980, rs150501885, and rs2275472. Among healthy donors, almost forty-percent had at least one CSF3R SNP, and two SNPs 
were found concomitantly in $32.96 \%$ of these donors, while only approximately $10 \%$ of healthy donors carried no SNPs. More details about the heterozygous genotype of the above SNPs and their distribution among healthy donors are listed in Table 2 and Fig 1, respectively.

\subsection{Association between donor CSF3R SNPs and engraftment of platelets and neutrophils}

CSF3R SNPs from healthy donors or autologous transplantation from patients with hematologic malignancies have now been shown to be related to peripheral blood stem cell enrichment ${ }^{[9]}$. Therefore, we wanted to determine whether the distribution of CSF3R SNPs in this study correlated with CD34 enrichment. The results showed that no SNPs were associated with CD34 enrichment.

CSF3R is a special receptor for G-CSF signaling and thus plays an important role in neutrophil development, survival and function ${ }^{[11]}$. We therefore explored the associations of CSF3R SNPs with neutrophils and PLT engraftment. The results showed that no associations were observed between the genotype frequencies of these six CSF3R SNPs and the engraftment of platelets or neutrophils (data not shown).

\subsection{Association of donor CSF3R SNPs with relapse and TRM}

Relapse occurred in 50 patients, including 38 patients who died of relapse and 12 patients who remained alive after treatment. None of the rs2275471, rs78861150, rs3917981, rs150501885, or rs2275472 polymorphisms were associated with the rate of relapse except for the rs3917980G/G or A/G genotype ( $\mathrm{p}=.082$ ) (Fig 2A). However, multivariate analysis including rs3917980 and disease risk showed no significant association $(\mathrm{HR}=1.6 ; 95 \%$ CI: 0.57-4.7, $\mathrm{p}=.366$ ) (Table 3 and Fig 5). Further subgroup analyses based on HLA loci showed that relapse had no association with rs3917980 in recipients receiving haplo-HSCT $(\mathrm{p}=.27)$, and none of those six SNPs were associated with relapse in the HLA-matched all-HSCT group (data not shown). Moreover, in this study, AML, MDS and CML were classified as myeloid leukemia, and ALL was classified as lymphocytic leukemia to elucidate whether there is a correlation between CSF3R SNPs and relapse in the above subgroup. The results showed that the rs3917980G/G or A/G genotype correlated with a lower incidence of relapse in the myeloid leukemia subgroup ( $\mathrm{p}=.047$ ) (Fig $2 \mathrm{~B}$ ).

Regarding the association between CSF3R SNPs and TRM, only the rs2275472T/C genotype was associated with a lower incidence of TRM (Fig 2C, $\mathrm{p}=.0031)$; however, further multivariate analysis showed no statistically significant association (Table 3). In subgroup analyses, this effect was confirmed in HLA-mismatched and HLA-matched recipients, as cumulative analysis showed that there was a significant association between the rs2275472T/C genotype and TRM ( $\mathrm{p}=.043$ and $\mathrm{p}=.032$ ) (Fig $2 \mathrm{D}$ and $2 \mathrm{E}$ ), while no associations were found in any of the rs2275471, rs78861150, rs3917981, rs3917980, and rs150501885 CSF3R SNPs (p>.05).

In view of the close correlation between TRM and SNPs, we aimed to understand the reasons for the increasing incidence of TRM caused by the $\mathrm{rs} 2275472 \mathrm{~T} / \mathrm{C}$ genotype. By analyzing correlations among posttransplantation infection, GVHD, platelet implantation, neutrophil implantation and SNPs, we found no evidence of an effect of the CSF3R SNP genotype on the incidence of aGVHD, and this result was further proven in all HLA-unmatched and HLA-matched patients. The genotypes of these six CSF3R SNPs did not influence the incidence of cGVHD and CMV (data not shown). However, the engraftment of PLTs was an independent protective factor against TRM ( $\mathrm{HR}=0.048,95 \%$ CI: 0.024-0.098, $\mathrm{p}<.001$ ) (Table 3). Cumulative analysis showed that good platelet implantation was associated with lower transplantation-related mortality $(\mathrm{p}<.000)($ Fig $2 \mathrm{~F})$.

\subsection{Association between donor CSF3R SNPs and patient survival}

In univariate analysis, we found that rs3917980 and rs2275472 polymorphisms in donors were associated with the LFS and overall survival of recipients (Table 3), while none of the other CSF3R SNPs in donors were associated with LFS or patient survival. Although CSF3R SNPs had statistically significant trends with LFS and OS, the biological results of LFS and OS associated with these the rs3917980 and rs2275472 genotypes were completely opposite. Patients receiving stem cells from donors with the rs $3917980 \mathrm{G} / \mathrm{G}$ or A/G genotype trended towards increased LFS (25.5\% versus $13.6 \%, \mathrm{p}=.016)$ and OS rates $(\mathrm{p}=.044)$ (Fig $3 \mathrm{~A}$ and $3 \mathrm{~B}$ ), while those with the rs2275472T/C genotype trended towards LFS rates ( $\mathrm{p}=.066$ ) (Fig $3 \mathrm{C}$ and 
3D). Further subgroup analyses based on HLA loci showed no relations between CSF3R SNPs and LFS and OS in HLA-matched groups ( $\mathrm{p}>.05$ ); however, patients receiving stem cells from HLA-mismatched donors with the rs3917980G/G or A/G genotype showed better LFS and a trend towards a higher OS rate ( $\mathrm{p}=.015$ and $\mathrm{p}=.098$, respectively) (Fig $3 \mathrm{E}$ and $3 \mathrm{~F}$ ). Notably, no association was found between the $\mathrm{rs} 2275472 \mathrm{~T} / \mathrm{C}$ genotype and OS in total HLA-unmatched recipients $(\mathrm{p}=0.42)$, and patients receiving stem cells from HLAunmatched donors with the rs2275472T/C genotype had no associations with incidence of LFS rates and OS $(\mathrm{p}>.05)$ (data not shown).

The Cox regression analysis showed that no significance was found for any CSF3R SNPs for predicting relapse, TRM, LFS or OS ( $\mathrm{p}>.05)$. The engraftment of PLTs was a protective factor for LFS and OS $(\mathrm{p}<.001$, Table 3$)$

Table 3 Univariate analysis for clinical outcomes of allogeneic stem cell transplantation

\section{Covariate Univariate analysis HR 95\% CI P}

\section{Relapse}

rs3917980 with or without $\mathrm{G} / \mathrm{G}$ or A/G 0.475 0.171-1.147 0.097

High risk vs standard risk 3.647 1.197-10.658 0.011

DTCD4A -0.004-0.321 0.056

DTLA 0.042-0.729 0.028

\section{TRM}

rs2275472 with or without T/C 3.77 1.416-9.631 0.004

High risk vs standard risk 2.023 0.539-6.400 0.1948

PLT 0.053 0.020-0.133<0.000

DTCD4A -0.289-0.003 0.045

\section{LFS}

rs2275472 with or without T/C 2.523 1.032-6.13 0.026

rs3917980 with or without G/G or A/G 0.432 0.191-0.903 0.018

High risk vs standard risk 3.740 1.307-11.201 0.007

PLT 0.038 0.009-0.118<0.000

\section{OS}

rs2275472 with or without T/C 1.785 0.725-4.310 0.192

rs3917980 with or without G/G or A/G 0.512 0.244-1.017 0.047

High risk vs standard risk 3.145 1.103-9.390 0.020

PLT $0.0340 .006-0.116<0.000$

ANC: Absolute neutrophil count; PLT: Platelet; DTCD4A: Absolute CD4 T cells of donors; DTLA: Absolute $\mathrm{T}$ cells of donors.

The rs3917980 genotype was associated with better LFS and OS in patients post allo-HSCT

As rs3917980 and rs2275472 SNPs showed a completely opposite effect on clinical prognosis after transplantation in univariate analysis and approximately $33 \%$ of the healthy donors carried two SNPs, we speculated 
whether the biological effects caused by the above SNPs would be weaker than those caused by a single SNP. Then, we divided all patients into the following four groups according to whether their corresponding donors contained two SNPs: rs3917980G/G or A/G genotype single-positive group, rs2275472 T/C genotype singlepositive group, double-positive group and double-negative group. Because the number of positive patients was only 4 out of 273 , we created a group with double-negative SNPs. Cumulative analysis of LFS and OS showed that recipients receiving grafts from healthy donors with the rs3917980 G/G or A/G genotype had higher LFS rates than those who received grafts from healthy donors with the rs22754272 T/C genotype ( $\mathrm{p}=.037$ ) (Fig 4A), while the LFS and OS rates of the wild-type genotype in the group with double-negative SNPs were between the values of the above groups, further proving that the rs $3917980 \mathrm{G} / \mathrm{G}$ or A/G genotype may be a strong protective factor for a better LFS; whoever no significant differences were found among the groups ( $\mathrm{p}=.14)$ (Fig 4B).

\section{Discussion}

In this paper, we would like to emphasize the following: (i) the CSF3R polymorphism rs2275472 in donors with the T/C genotype has a negative impact on the risk for TRM and LFS of patients post allo-HSCT; however, the rs3917980G/G or A/G genotype is considered a protective factor for LFS, and (ii) low platelet recovery identifies patients at greater risk for a poor prognosis.

The first observation that the two different SNPs of CSF3R in healthy donors have opposite effects on the clinical outcomes of recipients after allo-HSCT, which is perhaps not well documented in published studies. Previous studies have shown that CSF3R polymorphisms caused by mutation or alternative splicing lead to increased or decreased signaling cascades in response to G-CSF, leading to abnormal clinical outcomes ${ }^{[9]}$. CSF3R is widely distributed in hematopoietic cells, therefore, we hypothesize that as the donors' hematopoietic stem cells gradually chimerize in recipients, different SNPs are likely to mediate signal cascades, eventually leading to diverse clinical outcomes post allo-HSCT. Our further subgroup analysis results suggest that patients receiving grafts from healthy donors with the rs3917980 genotype have higher LFS rates than those receiving grafts from donors with the rs227542 genotype, suggesting that CSF3R site screening in healthy donors may be used as an indicator to predict the outcome of patients. However, more research is needed. No one has discussed the potential effects of these two SNPs on clinical outcomes so far. Regarding why the CSF3R SNP site in the intron region can affect the prognosis of transplantation, we speculated that this effect may be caused by the intron region affecting mRNA expression by changing the transcription efficiency, splicing or maturation of $\mathrm{mRNA}^{[12]}$. Our next step may focus on verifying whether SNP site can affect the transcription and protein expression of the CSF3R gene by interfering with the intron or exon zone.

The second result of our study was that multivariate analysis showed that poor platelet recovery was a negative predictor of transplant-related mortality, leukemia-free survival and overall survival. This has been elucidated in various reports ${ }^{[13,14,15]}$. The reason for the above results may be that poor platelet implantation is related to the occurrence and development of acute and chronic GVHD, which leads to high TRM, low OS rates and better LFS $^{[16]}$. A previous study elucidated that CSF3R mutations in AML patients were associated with significantly lower platelet counts ${ }^{[17]}$. In this study, we found no association between platelet implantation and SNPs of CSF3R, and further studies are still needed to explore the clinical characteristics of patients receiving grafts from healthy donors with different CSF3R SNPs due to the relatively small number of participants included in this study.

G-CSF is an important regulator cytokine for the development and function of neutrophils, and most studies have previously proven that mutations of CSF3R lead to increased or decreased numbers of neutrophils because of disorder of the CSF3R signaling cascade ${ }^{[18]}$. However, regarding the correlation of CSF3R SNPs with granulocytes, no reports have been found. In this study, we found no association between CSF3R SNPs and neutrophil recovery. Further large prospective studies are needed.

In summary, we have reported that two CSF3R polymorphisms in healthy donors predicted completely opposite results in recipients, indicating that a comprehensive assessment of donor CSF3R may be valuable 
in patients receiving allo-HSCT. Obviously, these data must be validated in larger patient cohorts, and the pathophysiological functionality of these polymorphisms should be further elucidated in mechanistic in vivo and in vitro models.

\section{References}

[1] Alvarez P J, Carrillo E, Velez C, et al. Regulatory systems in bone marrow for hematopoietic stem/progenitor cells mobilization and homing[J]. BioMed Research International, 2013: 312656-312656.

[2] Pelus L M. Peripheral blood stem cell mobilization: new regimens, new cells, where do we stand[J]. Current Opinion in Hematology, 2008, 15(4): 285-292

[3] Horan T, Wen J, Narhi L,et al.Dimerization of the extracellular domain of granuloycte-colony stimulating factor receptor by ligand binding: a monovalent ligand induces 2:2 complexes[J]. Biochemistry. 1996; $35: 4886-4896$

[4] Futami M, Zhu QS, Whichard ZL,et al.G-CSF receptor activation of the Src kinase Lyn is mediated by Gab2 recruitment of the Shp2 phosphatase[J]. Blood. 2011; 118:1077-1086.

[5] Lantow M, Sivakumar R, Zeumer L, et al. The granulocyte colony stimulating factor pathway regulates autoantibody production in a murine induced model of systemic lupus erythematosus $[\mathrm{J}]$. Arthritis Research \& Therapy, 2013, 15(2): 1-14.

[6] Mehta H, Futami M, Glaubach T, et al. Alternatively spliced, truncated GCSF receptor promotes leukemogenic properties and sensitivity to JAK inhibition[J]. Leukemia, 2014, 28(5): 1041-1051.

[7] Hunter MG, Avalos BR. Deletion of a critical internalization domain in the CSF3R in acute myelogenous leukemia preceded by severe congenital neutropenia[J]. Blood. 1999; 93:440-446.

[8] Ward A C, Van Aesch Y M, Schelen A M, et al. Defective internalization and sustained activation of truncated granulocyte colony-stimulating factor receptor found in severe congenital neutropenia/acute myeloid leukemia[J]. Blood, 1999, 93(2): 447-458.

[9] Boguniakubik K, Gieryng A, Gebura K, et al. Genetic variant of the G-CSF receptor gene is associated with lower mobilization potential and slower recovery of granulocytes after transplantation of autologous peripheral blood progenitor cells[J]. Cytokine, 2012, 60(2): 463-467.

[10] Camurdanoglu B Z, Esendagli G, Ozdemir E, et al. The effect of granulocyte colony stimulating factor receptor gene missense single nucleotide polymorphisms on peripheral blood stem cell enrichment[J]. Cytokine, 2013, 61(2): 572-577.

[11] Dwivedi P, Greis K D. Granulocyte colony-stimulating factor receptor signaling in severe congenital neutropenia, chronic neutrophilic leukemia, and related malignancies[J]. Experimental Hematology, 2017: 9-20.

[12] Wang H, Feng L, Niu D, et al. Relationship between mRNA stability and intron presence[J]. Biochemical and Biophysical Research Communications, 2007, 354(1): 203-208.

[13] Akahoshi Y, Kimura S, Gomyo A, et al. Delayed platelet recovery after allogeneic hematopoietic stem cell transplantation: Association with chronic graft-versus-host disease and survival outcome[J]. Hematological Oncology, 2018, 36(1): 276-284.

[14] Dominietto A, Raiola A M, Van Lint M T, et al. Factors influencing haematological recovery after allogeneic haemopoietic stem cell transplants : graft-versus-host disease, donor type, cytomegalovirus infections and cell dose[J]. British Journal of Haematology, 2001, 112(1): 219-227.

[15] Moneib H, Hafez H, Abdalla A, et al. Day +100 Platelet Count Predicts Survival After Allogeneic Hematopoietic Stem-Cell Transplantation in Children With Hematologic Malignancies[J]. Clinical Lymphoma, Myeloma \& Leukemia, 2019, 19(5). 
[16] Pulanic D, Lozier J N, Pavletic S Z, et al. Thrombocytopenia and hemostatic disorders in chronic graft versus host disease[J]. Bone Marrow Transplantation, 2009, 44(7): 393-403.

[17] Su L, Gao S, Tan Y, et al. CSF3R mutations were associated with an unfavorable prognosis in patients with acute myeloid leukemia with CEBPA double mutations[J]. Annals of Hematology, 2019, 98(7): 16411646 .

[18] Fleischman A G, Maxson J E, Luty S B, et al. The CSF3R T618I mutation causes a lethal neutrophilic neoplasia in mice that is responsive to therapeutic JAK inhibition[J]. Blood, 2013, 122(22): 3628-3631.

Table 1 Subject characteristics and outcomes $(\mathrm{N}=273)$

AML: Acute myeloid leukemia; ALL: Acute lymphoid leukemia; MDS: Myelodysplastic syndrome; cGVHD: Chronic graft versus host disease; LFS: Leukemia-free survival; TRM: Transplantation-related mortality.

Table 2 Different donors' CSF3R SNPs and the percentages of polymorphic sites analyzed in this study.

SNPs: single-nucleotide polymorphisms.

Figure 1 The frequency of CSF3R SNPs distributed among the recipients with different diagnoses $(\mathrm{n}=273)$. The inner cycle was composed of disease categories in this study, and the outer rings indicated the proportion of different cumulative CSF3R SNPs in the inner circle.

Figure 2 The associations of CSF3R SNPs with TRM and relapse.

Figure 3 The association between overall survival and LFS probabilities and rs3917980 and rs2275472 polymorphisms.

Figure 4 Analysis of LFS and OS among CSF3R SNPs.

Figure 5 Multifactor Cox regression of relapse, TRM, LFS and OS. 


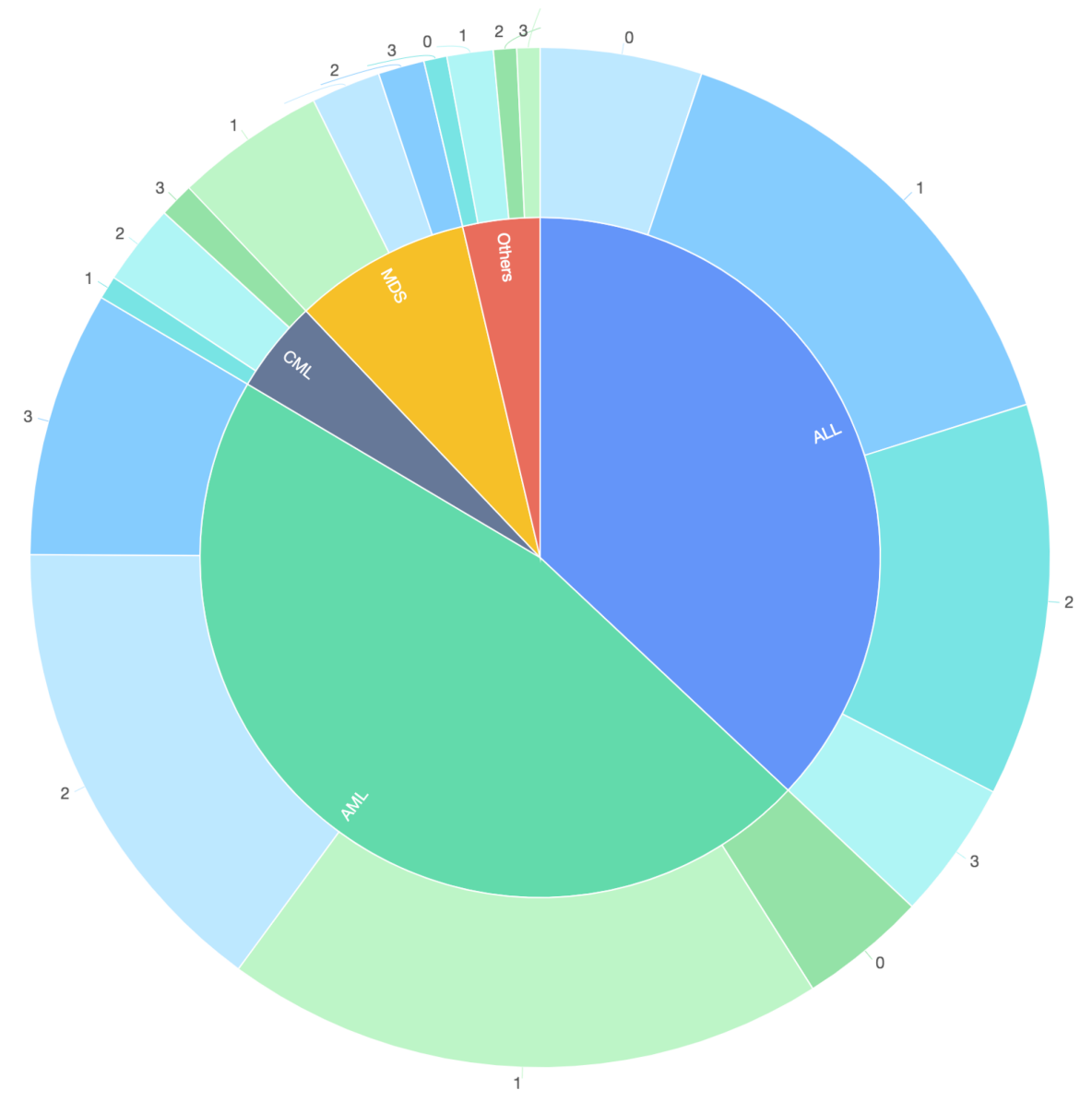


A

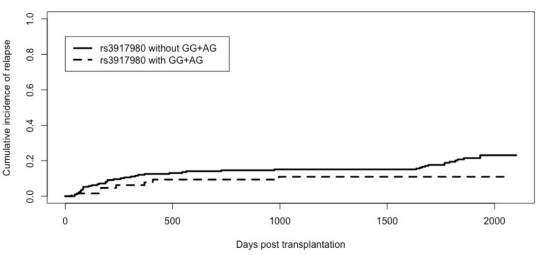

C

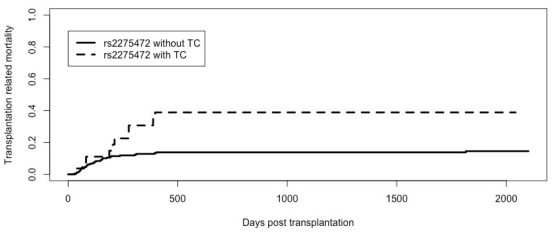

E

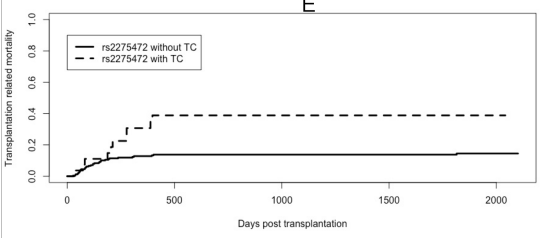

A

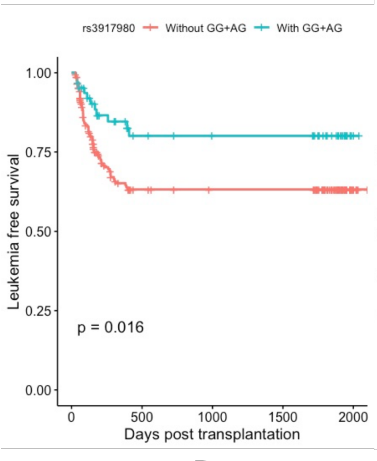

D
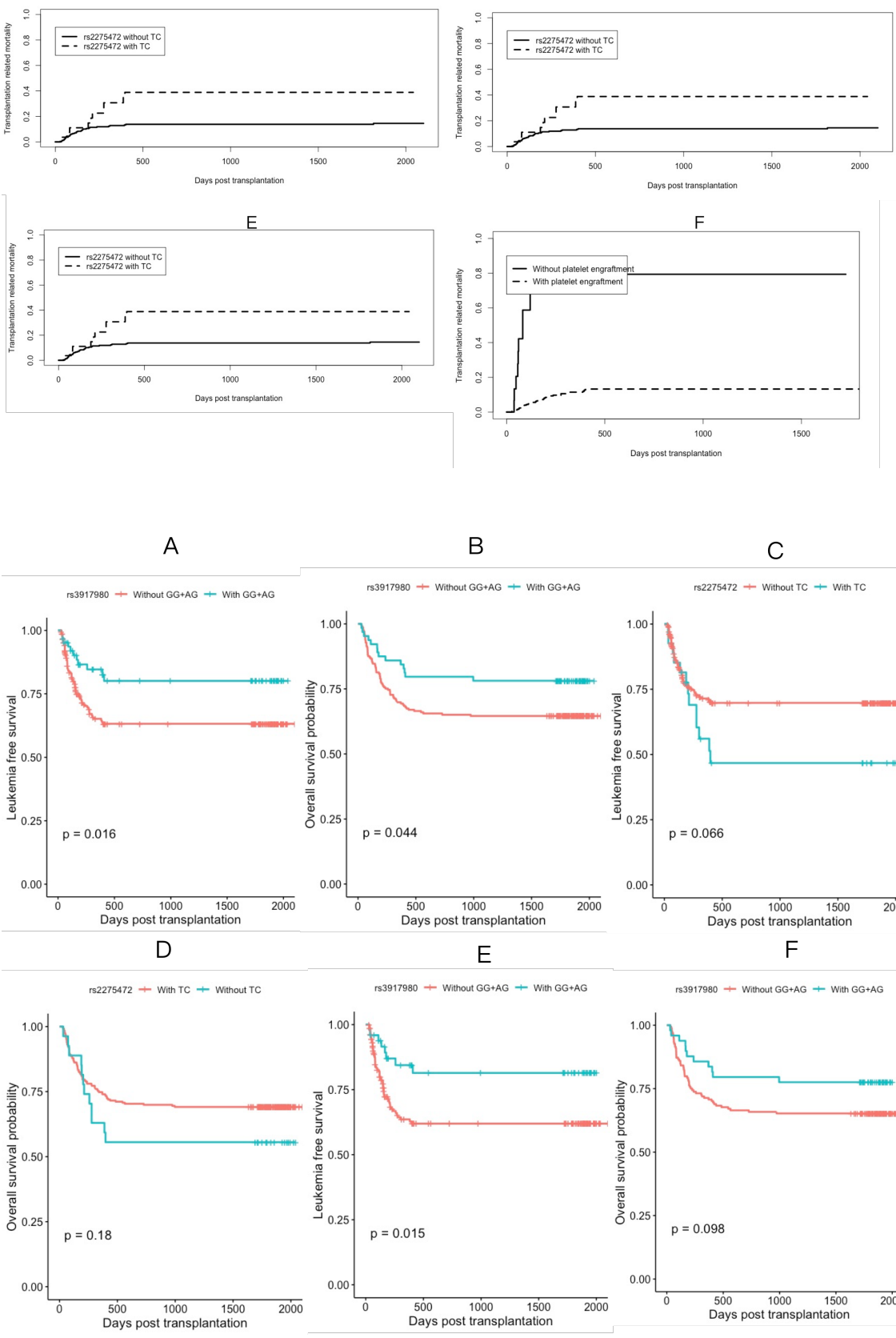

F

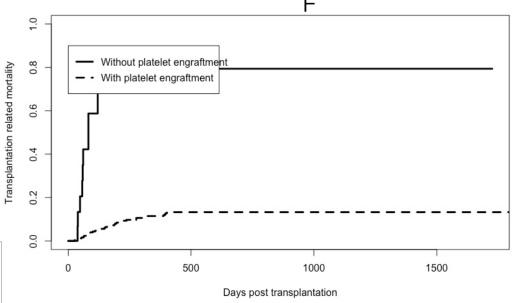

B

C
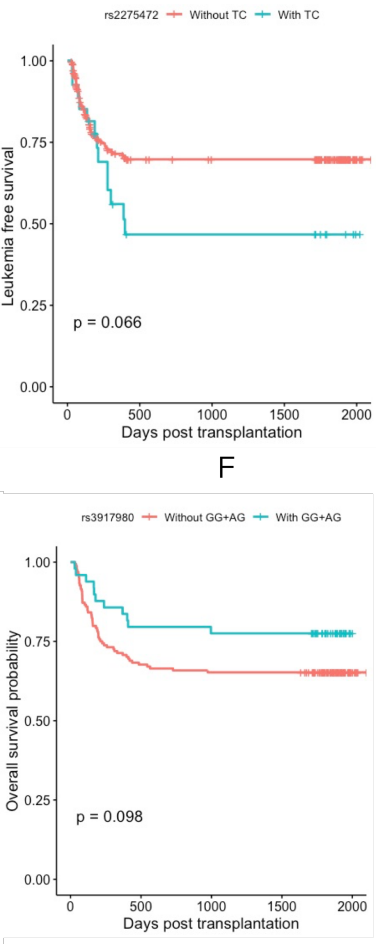
A

Strata + rs $3917980+$ rs $2275472+$ the rest

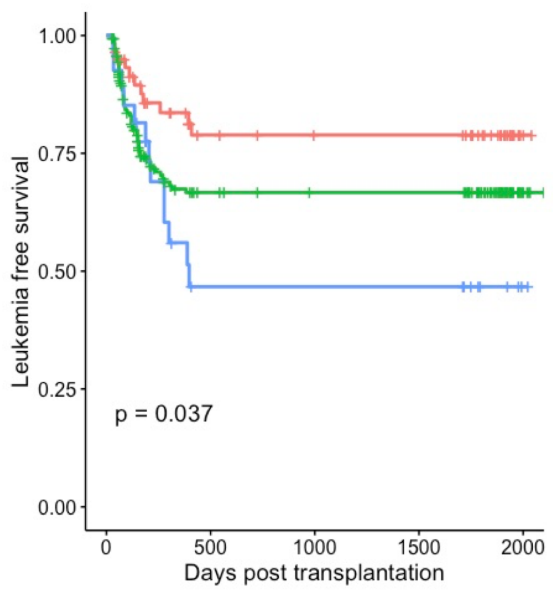

B

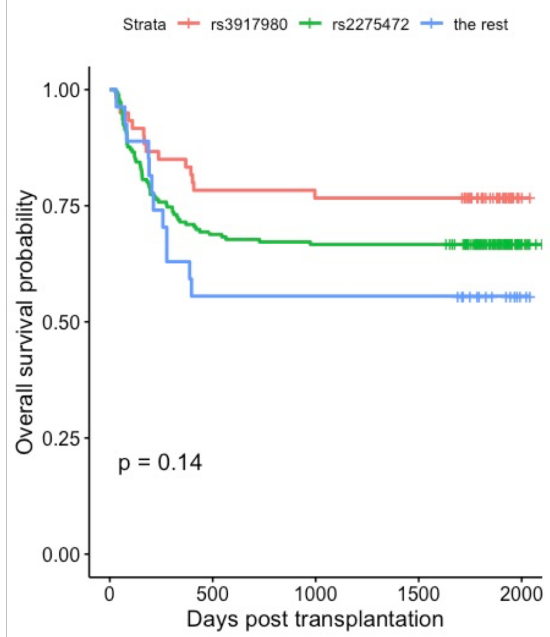

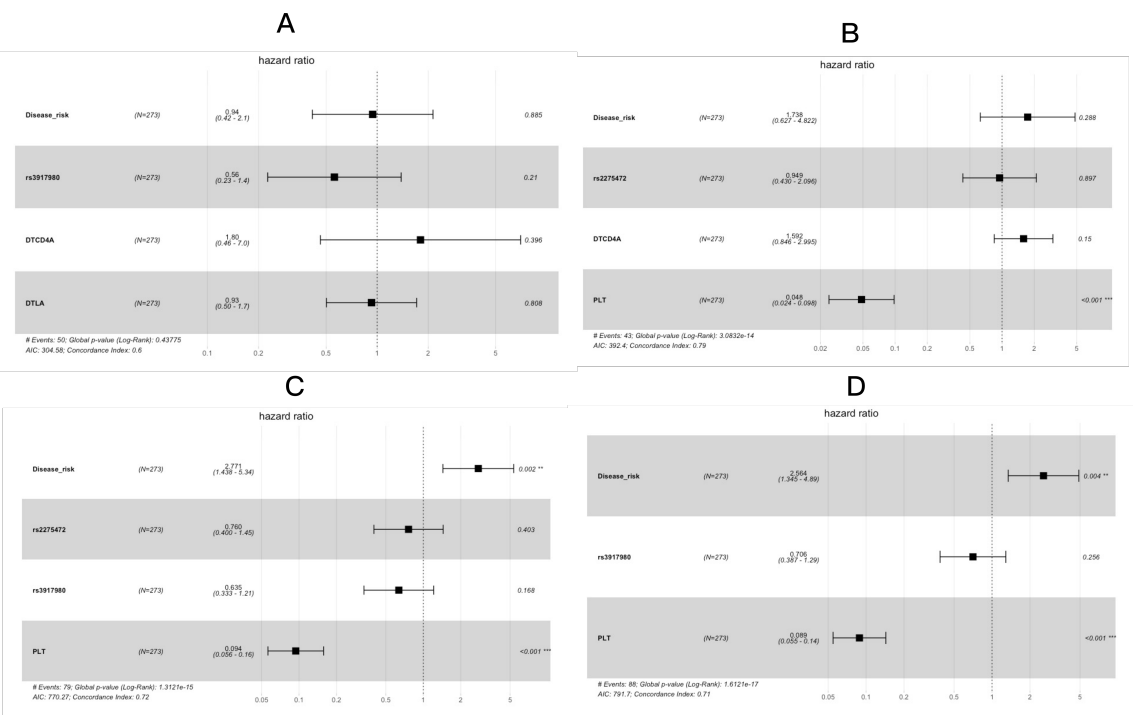

\section{Hosted file}

Table CSF3R.docx available at https://authorea.com/users/338028/articles/463991-donor-csf3rwith-the-rs3917980a-g-or-g-g-genotype-is-correlated-with-better-leukemia-free-survivalafter-allogenic-hematopoietic-stem-cell-transplantation 